\title{
Spaces of Information Modeling, Action, and Decision Making
}

\author{
Guy De Tré and Wouter Van Acker
}

\begin{abstract}
Nowadays, tremendous information sources are preserved, ranging from those of a traditional nature like libraries and museums to new formats like electronic databases and the World Wide Web. Making these sources consistent, easily accessible, and as complete as possible is challenging. Almost a century ago, people like Paul Otlet were already fully aware of this need and tried to develop ways of making human knowledge more accessible using the resources and technology available at that time. Otlet's ideas about a Universal Network of Documentation and the Universal Book are clear examples of such efforts. Computer science currently provides the means to build digital spaces that consist of (multimedia) information sources connected through the Internet. In this article, we give a nontechnical overview of the current state of the art in information management. Next, we focus on those aspects of Otlet's work that deal with the organization of knowledge and information sources. Then we study the potential connections between Otlet's work and the state of the art of computerized information management from a computer scientist's point of view. Finally, we consider some of the problems and challenges that information management still faces today and what computer science professionals have in common with, and can still learn from, Otlet and his work.
\end{abstract}

\section{INTRODUCTION}

Information management, despite its fast development, faces important technological problems and significant challenges. One of those challenges concerns the need to bridge the gap between how information and knowledge are perceived, used, communicated, and visualized by humans and how they are represented, stored, and managed with computer

LIBRARYTRENDS, Vol. 61, No. 2, 2012 ("Information and Space: Analogies and Metaphors," edited by Wouter Van Acker and Pieter Uyttenhove), pp. 304-324. (C) 2012 The Board of Trustees, University of Illinois 
systems. Indeed, on the one hand, current computing techniques are based on rigid data(base) models and knowledge-base models, which can be seen as a collection of rules that together prescribe how data should be structured and handled conceptually in a computer system and how the correctness of these data can (as far as possible) be guaranteed. On the other hand, human beings communicate by using natural language, which does not follow the rules of the language used by computer systems. Hence, in order to "translate" information and knowledge as used and communicated by humans to their computerized counterparts, the rules of a selected data(base) model or knowledge-base model must be used. Such a translation process almost always causes an unfavorable information loss. Stored digital data are visualized and presented to users through prebuilt human-computer interfaces that can be specifically fine-tuned to the individual needs of the user. So, there is a clear distinction between how data are modeled and handled conceptually by the computer system and how these data are presented to the users.

Because of this gap, current conceptual models still face the problem of not being able to model human information and knowledge without information loss. In general, information loss can be caused by all kinds of data imperfections such as imprecision, uncertainty, vagueness, incompleteness, and inconsistency. To reduce information loss, data, information, and knowledge must be modeled as adequately and as naturally as possible (Korth \& Silberschatz, 1997). This is especially important because correct data manipulation, querying, and decision making are not possible without correct, adequate data.

The problems of correct and adequate data(base) modeling, as encountered in computer science, are not new. At the beginning of the twentieth century, Paul Otlet (1868-1944) struggled with similar challenges. During his professional life, Otlet studied—on a theoretical, organizational, and technical level—how information and knowledge could be made accessible for everybody. He consequently faced the problem of structuring, classifying, and representing information and knowledge in a universal way, preferably without data loss. It is interesting to observe that Otlet's conceptual models and proposals for classifying, filing, and handling information in (virtual) knowledge spaces have more in common than one would think with the digital spaces of current computer-based information systems.

In this article, we study from a computer scientist's point of view the analogies between Otlet's knowledge spaces and current digital spaces. More specifically, we investigate what modern information- and knowledge-management techniques have in common with the proposals presented by Otlet almost a century ago. We examine the extent to which this work could be an inspiration for the development of more effective techniques to structure, visualize, and handle information and to prevent information loss in future information systems. 


\section{The Organization of Digital Spaces}

Digital spaces presently allow us to manage and share digital information that is stored on computer systems scattered all over the world and connected through the Internet. Advanced querying and informationretrieval and data-mining techniques allow the exploitation of tremendous data collections and provide users with information and knowledge to support their decision making and to steer and verify their actions. The left triangle depicted in figure 1 shows the subcategories in which digital spaces of information modeling, action, and decision making are usually organized by computer scientists. The right triangle in the figure shows the technologies and tools used to handle these subcategories. The representation is triangular to reflect the quantity of available facilities. There are far more techniques to deal with structured, semistructured, and unstructured data than there are techniques to deal with actions. The triangles also reflect the historical development of the techniques: at the base, we have the oldest, best-known, and best-developed technologies. The more we move to the top, recent techniques increase. The dotted horizontal line represents the current practical state of the art. Technologies below this line are commonly and widely used in companies, while technologies above the line are not so commonly used in practice and definitely need further development.

\section{Structured, Semistructured, and Unstructured Data}

At the base of the left triangle are the structured, semistructured, and unstructured data sources. In the case of structured data, given facts are typically represented by character strings or numbers and structured in record types, tables, or class structures. Unstructured data typically consist of long byte sequences and are the result of a digitization process. Digitized photographs and audio and video recordings are examples of unstructured data. Another type of unstructured data concerns very long character sequences that result from text processing or optical character recognition (OCR) applications. In semistructured data, both structured and unstructured components are combined. XML and HTML documents that are used for the construction of web pages are examples of semistructured data. Structured, semistructured, and unstructured data are typically conceptually modeled in a one-dimensional record structure or two-dimensional table structure and managed with file systems or more advanced database systems (Elmasri \& Navathe, 2007).

\section{Multidimensional Data}

Data stored in databases are intended and used to support real-time needs and processes of an individual or a company. However, database systems are not well equipped for complex analytical purposes. Complex data analysis often involves different aspects of the same data. For example, if a company wants to analyze its sales, it might do this by considering 


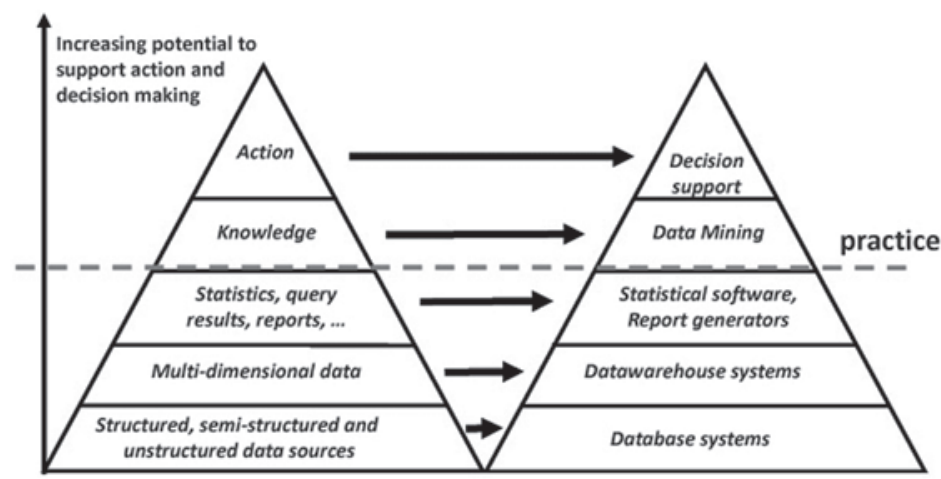

Figure 1. Organizing digital spaces.

the time, location, and category of the sales transactions. Or it might consider the profiles of its customers, or even take into account the presales representative that was involved in the transaction. Therefore, a new kind of information-management system, the data-warehouse system, has been introduced (Kimball \& Rose, 2002). Multidimensional hypercube structures are typically used in data-warehouse systems. The cells of such a cube contain the facts that must be analyzed (e.g., sales), whereas the dimensions of a cube are used to model the different aspects of a fact. As such, the dimensions for sales analysis could be the time, location, product category, customer profile, and presales profile of sales transactions. Dimensions typically have a hierarchical structure. For example, the location could be registered at country, region, city, street, and shop levels.

Statistics, Query Results, and Reports

Databases are queried by means of a query language that is provided by the database system. The query language prescribes how users can compose their ad hoc queries. Analysis of data warehouses is made with online analytical processing (OLAP) tools or statistical tools. The query or data analysis results need to be presented to the users. Automatic report generators can generate reports that are easy to interpret by those who are involved in decision making.

\section{Knowledge}

Human knowledge is usually represented in the form of so-called knowledge rules or in the form of patterns. An example of a knowledge rule is: "If a customer is middle-aged, married and has a well-paid job, then he or she has a low-risk loan profile." An example of a pattern is: "Customers who buy products A and B will most likely also buy product C." Knowledge rules and patterns can be managed and stored in a knowledge-based system. Such systems allow for deriving new data by applying knowledge 
rules and patterns to known data. There also exist special computation techniques that allow for extracting new knowledge rules or patterns from huge data collections. Such techniques are usually based on statistical correlation and are called data-mining techniques (Dunham, 2006). These techniques are used to "mine" the data for hidden, previously unknown knowledge.

\section{Action}

All data and knowledge present in an information system can be used to support and steer decision making. Decision support-system software is specifically designed to analyze and evaluate data to provide users with motivated arguments to choose the best-suited option from a set of alternatives. Together with data-querying and analysis techniques, decision support tools offer users the information that they may require to verify their actions.

\section{The Organization of Otlet's Knowledge Spaces}

In the precomputer age, Otlet, a Belgian bibliographer, encyclopedist, and founding father of the discipline of "documentation," already faced the problems and challenges of collecting and organizing human information and knowledge with the aim to make it globally accessible to everybody. Although his work was mainly based on labor-intensive, mechanical, and manual approaches that were constrained by the technological limitations of his time, one can look at his knowledge spaces from a computer scientist's point of view and study the extent to which his ideas, concepts, and proposals are still valid, useful, or applicable in more recent digital spaces.

Otlet's pyramidal model of the organization of documentation, for example, as shown in figure 2, is conceptually continuous with the pyramidal model of digital spaces that we presented earlier. The sequence of Library, Bibliographic Repertory, Documentary Encyclopedia, and Synthesis reflects the historical development of these techniques but also mirrors the idea that, ultimately, all sorts of documentation must be organized and synthesized in such a way that the information it contains is immediately available to support action and decision making. The emphasis on the application of all data for the support of action and decision making was fundamental to what Otlet defined as "documentation" as early as 1907:

Documentation is today defined as the implementation of all written or graphic sources of our knowledge, as constituted by documents or any kind of printed texts in particular. These documents comprise everything that represents or expresses with the aid of signs of all kinds (writing, pictures, schemas, and symbols), an object, a fact, or an impression ... The aim of documentation is to rapidly and easily furnish all researchers, whatever their level of knowledge or culture, 
with the study materials that are the sum of universal experience, and with detailed pieces of information on particular points of interest. (Otlet, 1907, pp. 7-8)

Otlet's approach to documentation and information was innovative in its focus on the coordination and codification of content and on the analysis and summarization of information as contained in documents. For Otlet, it was fundamental that the organization and dissemination of documentation and information be made in a universal, and thus standardized, manner. From a computer scientist's point of view, his concept of the Universal Network of Documentation and his concept of the Universal Book can be considered most important. Both are briefly described next.

\section{The Universal Network of Documentation}

In 1895, Otlet and his colleague Henri La Fontaine started to develop their Universal Decimal Classification (UDC) system for the organization of documents and all other sources of information, which later became the most widely used classification system in the world and has been adopted by the International Organization for Standardization (ISO). The UDC can be seen as a multidimensional classification in which each source is assigned a category, denoted by a numerical code, in each dimension. The classification code corresponding to a source is then obtained by appropriately concatenating the assigned category codes. The dimensions considered in the UDC are materials (matières), relationship (relation), location (lieu), time (temps), form (forme), and language (langue). In figure 3, an illustration of the different facets of the UDC is presented.

On the basis of the UDC and a central index card-and-cabinet system, Otlet and La Fontaine envisioned a system that would enable people to conduct comprehensive mechanical bibliographical searches in the central Universal Bibliographic Repertory by mailing their query in conceptually almost the same way as people today resort to online searching in a distant bibliographic database. In 1925, Otlet formulated the idea to connect principal documentation offices and libraries in a "Universal Network of Documentation" centralized around a "Document Super-Centre" (Otlet \& Goldschmidt, 1925). In Traité de Documentation (1934), he defined the concept of the "Universal Network of Documentation" in the following terms:

The Network must link together, by whatever means, the centres of production, distribution, and use, regardless of specialization and place. In practical terms, it is the matter of every producer who has a fact to make public, or a proposition to present or to defend; every user who needs information for the development of his theoretical or practical work; and every person, ultimately, being able to get hold of what is available to them with the minimum of effort and the maximum in terms of assurance and reward. (p. 415) 


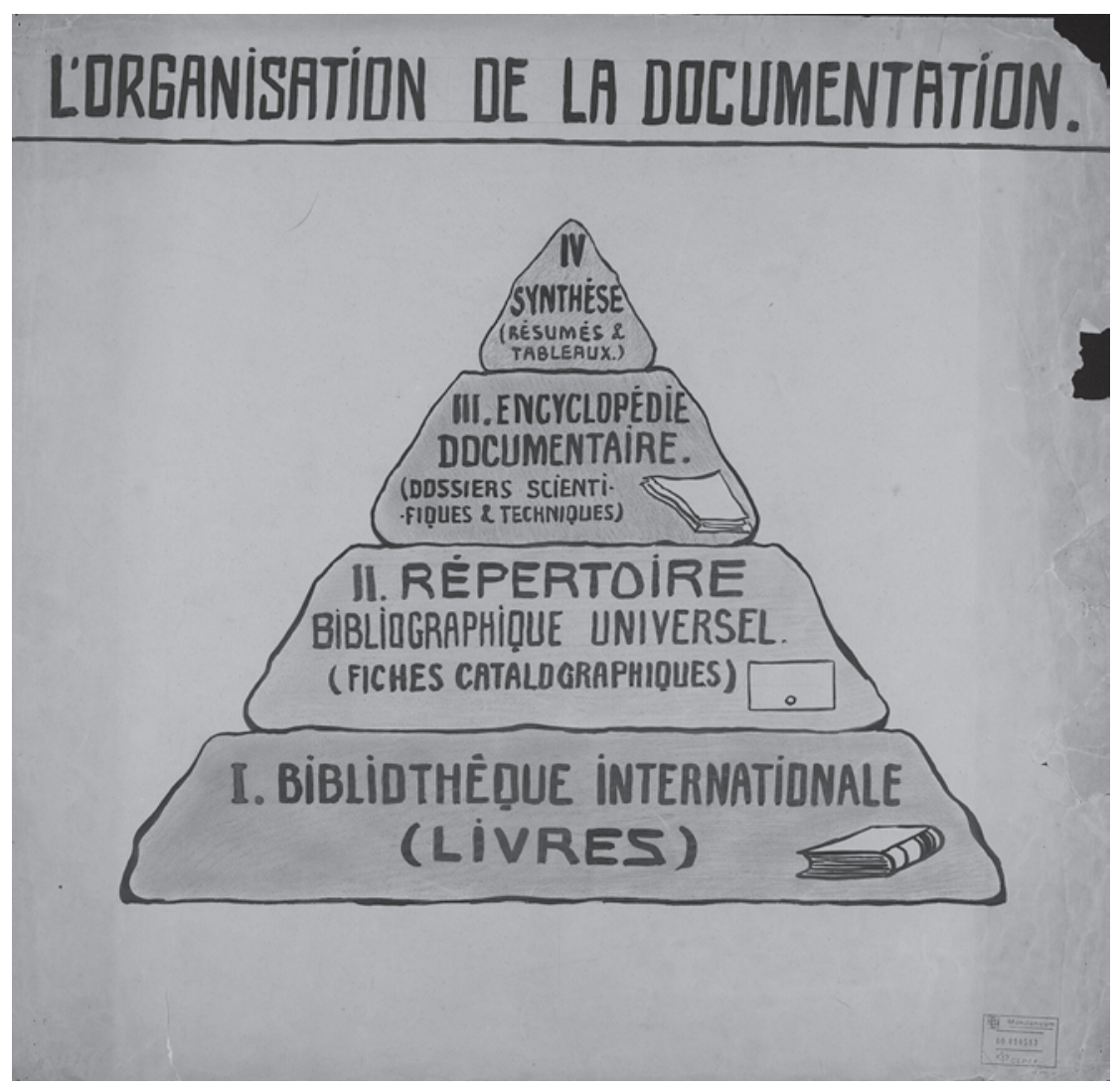

Figure 2. Paul Otlet. The Organization of Documentation (Mundaneum, EUM, fonds Affiches, doc nr. 00 010513).

In a similar way to the Internet-the global computer network that provides access to the World Wide Web-the Universal Network of Documentation would be a global network of communication, cooperation, and exchange, providing access to a vast collection of documentation (articles, books, audio files, images, and even movies). Unlike the Internet, however, the Universal Network of Documentation would centrally control the implementation of standards by each constituent of the network.

\section{The Universal Book}

Otlet stated that documentation should also have as its objective to analyze and summarize documents and to codify their content. It should merge the original data (facts, theories, methods, and projects) into the uniform and impersonal framework of a sort of Universal Book. The Universal Book would be a synthesis of all that had been written on a 


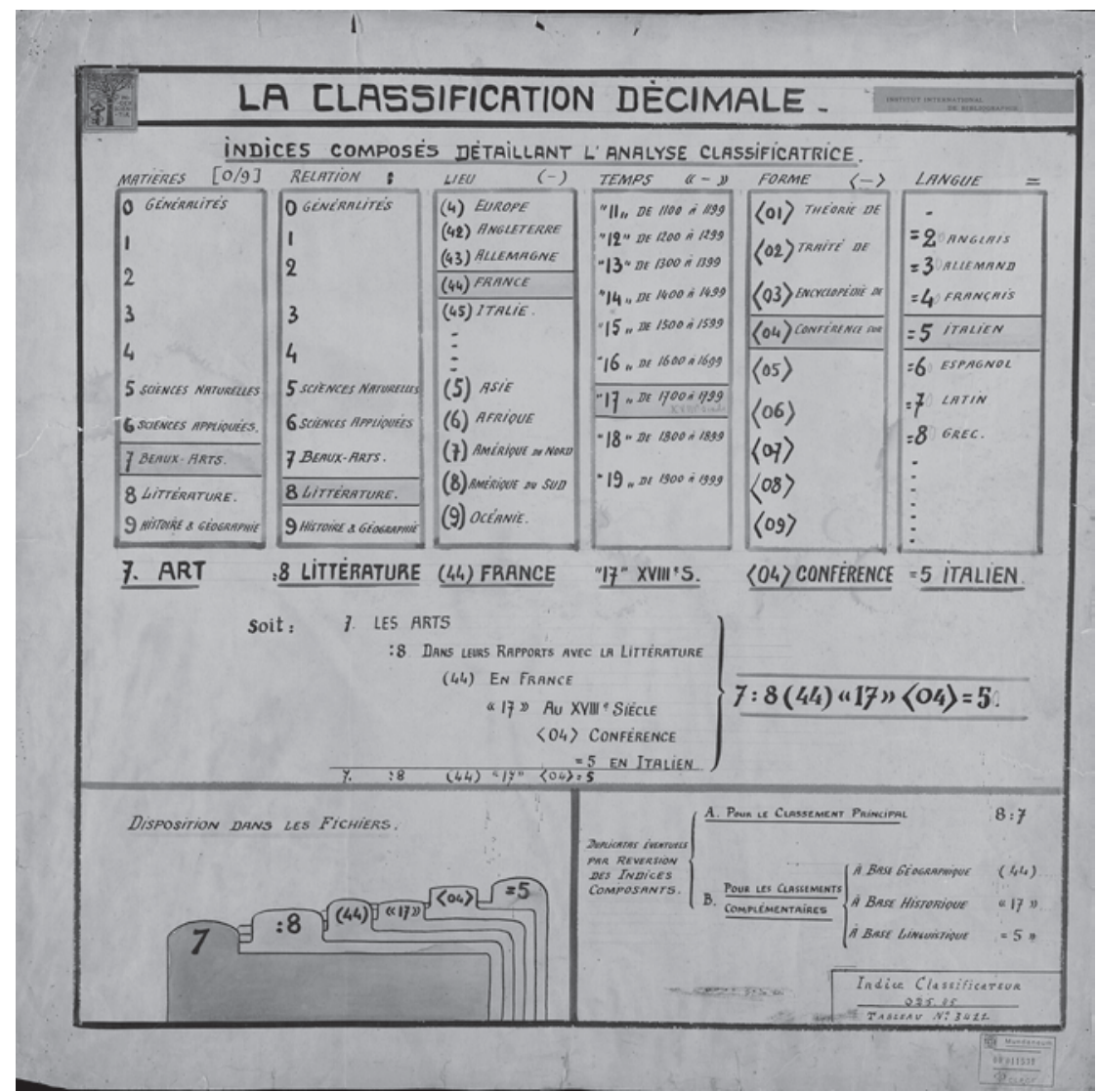

Figure 3. Illustration of the different facets of the UDC (Mundaneum, EUM, fonds Affiches, doc nr. 00 011531).

particular topic or discipline, and as such would be the necessary preparatory and intermediary step to build, from the multiplicity of books and documents, the Permanent World Encyclopedia.

Similar to Wikipedia-the free encyclopedia of the Internet-Otlet's Permanent World Encyclopedia aimed to collect and synthesize all information in the world. However, unlike Wikipedia, which uses hyperlinks to browse through content, the Permanent World Encyclopedia and the Universal Books of which it would be composed would use the UDC system to support efficient access and use. In the Universal Book, each registered fact would be assigned a classification code, based on the UDC, which would situate the fact in relation to a wider set of facts and thus make the "place" of the fact clearly visible in the classification's hierarchy. 


\section{Digital Spaces Versus Otlet's Knowledge Spaces}

While studying "documentation," Otlet was in essence dealing with the question of finding efficient solutions for the universal organization of data, information, and knowledge. The same concern still occupies all researchers dealing with current digital information-management systems. Hence, it is worth exploring the links between Otlet's knowledge spaces and current digital spaces.

Many aspects of digital spaces can be considered in a comparative study. Among the most important ones, we identified database models, data-warehouse models, multimedia, and distributed information systems. All of them will be discussed next. The objective of this discussion is twofold: to clarify the current state of the art of these aspects of digital spaces and to reveal the links with Otlet's work as seen from a computer scientist's point of view.

\section{Database Models}

Database models prescribe, among other things, how data in a database have to be structured and manipulated. Today, databases are most frequently based on the object-relational database model, which is an extension of the relational database model originally presented by Codd (1970). Central in the model is the concept of a relation, a two-dimensional structure, with a unique name. Relations are usually represented by tables. The columns of a table, representing the characteristics (or attributes) of the table, form its first dimension, whereas its rows, representing the content of the table, form the second dimension. A relational database consists of a set of tables, which can be related to each other. This implies that a row in one table can be coupled with one or more (related) rows in another table. Hence, the relationships between tables in a relational database generally have a one-to-many cardinality. The table names, column names, and column data types are examples of metadata describing the schema (and hence part of the semantics) of the database. In figure 4, a simple relational database to store information about paintings is presented.

Object-relational databases are excellently suited to handle tabular information, but not all available information can be reshaped easily into a tabular form. Hence, more advanced database models are required. Looking for links with Otlet's work, we mainly identify that the need to make a clear distinction between data and metadata in databases is also present in Otlet's knowledge spaces. Indeed, the UDC and a central index card-and-cabinet system can be seen as metadata systems that allow one to efficiently identify and find the documents one is looking for. It is also remarkable that Otlet, in his first proposals on handling documentation, already considered models that have more than two dimensions and hence are more complex. Was he already aware of the limitations of tabular representations? 


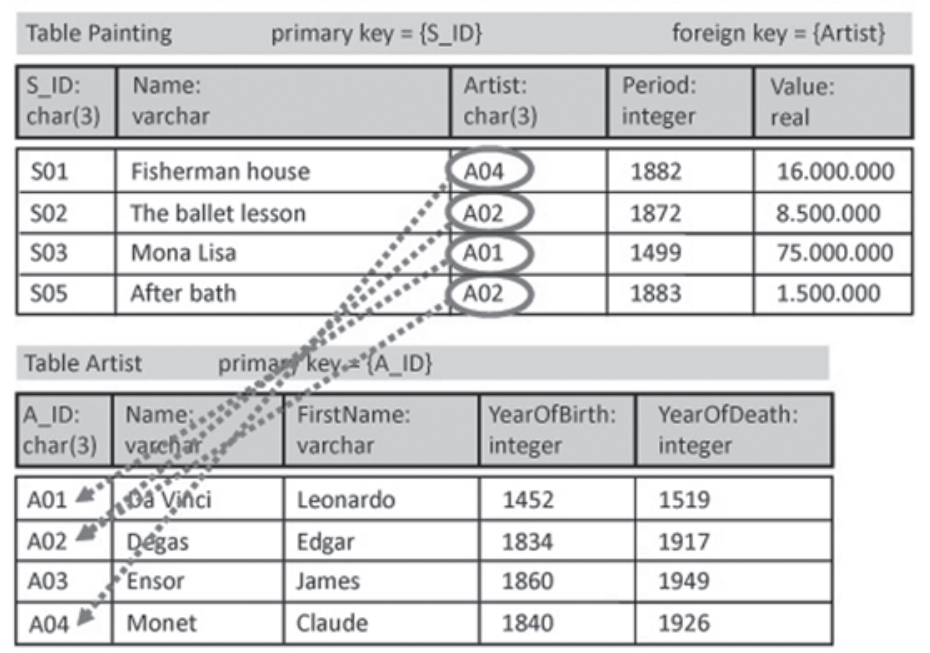

Figure 4. An example of a relational database.

\section{Data-Warehouse Models}

It is currently also generally recognized that the object-relational database model is not well suited for analytical purposes. This is mainly because the model lacks efficient facilities for automatically handling the different contexts (or dimensions) in which data can be approached. To address these shortcomings, Bill Inmon (1992) introduced the multidimensional data-warehouse model. Central in the data-warehouse model is the vision that data analysis is mostly based on the analysis of facts, where facts are characterized by a number of properties. Each property specifies a specific aspect of the facts and allows them to be qualified.

Multidimensional data cubes, also called hypercubes, are used to model facts and their properties. A data warehouse then consists of a number of such hypercubes. In the simplest approach, each hypercube is used to model information related to a single fact. Data about the fact are then stored in the cells of the hypercube, whereas each property of the fact is modeled by a dimension of the cube. A fact with $n$ properties will thus be represented by an $n$-dimensional hypercube. At the left side of figure 5 , an example of a three-dimensional hypercube is given. This cube is used to model the quantity of sold products, considering only the time, location, and product aspects of these sales.

The data-warehouse model also provides adequate operators for handling hypercubes. These operators are commonly known as the OLAP operators. Examples are the operators to rotate a cube, to roll-up and drill-down to another level of detail, to select cells on the basis of a given criterion, and to sort the cells in a hypercube. Using the OLAP operators, 

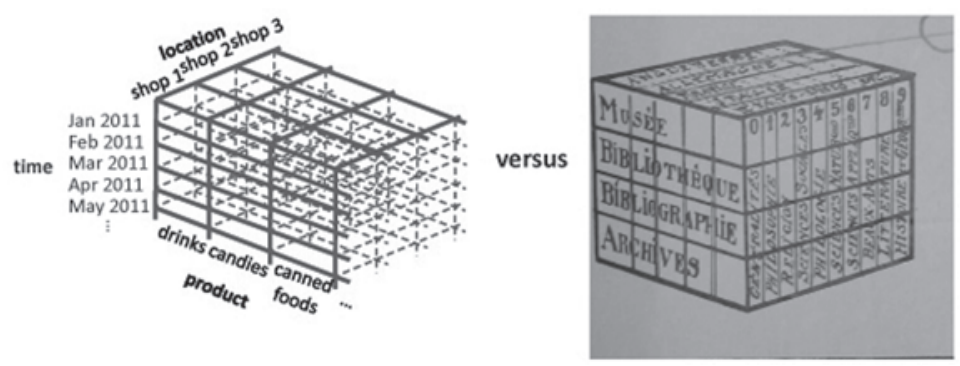

Figure 5. A three-dimensional hypercube versus Otlet's three-dimensional data-cube model (Image on left: author. Image on right from Paul Otlet, Tables et Diagrammes [brochure], in Mundaneum, EUM 10.).

users can analyze data warehouses in a visual way. The results of analytical operations are immediately reflected in a new hypercube that is presented to the user. As such, the impact of analytical operations can be easily observed and interpreted.

While dealing with the problem of how to structure and organize documentation, Otlet also studied and developed several generic models to situate registered facts in relation to a wider set of facts and to find the UDC code that leads to the "address" of the knowledge resources that correspond to these facts. In one of these models, Otlet proposed to structure information in "mechanical" cubes as presented at the right side of figure 5.The central idea behind this cube model was to develop a threedimensional cube index for the "universe of knowledge," which allows for efficiently retrieving documentation based on the values of dimensions of the UDC that have been selected for the construction of the cube. The dimensions used in the cube of figure 5 are the location with which the documentation is connected (country), the category of the documentation (general information, philosophy, religion, social sciences, philology, nature science, etc.), and the type of documentation source (museum, library, bibliography, archive, etc.).

It is amazing to observe how much Otlet's cube model has in common with the data-warehouse hypercube model that was invented about fifty years later and is now widely used in information systems. Indeed, in the data-warehouse model, one also works with dimensions that model certain characteristics (or properties) of facts. The dimensions allow for efficiently tracking and analyzing information about given properties of this factual data. In Otlet's mechanical-cube model, the facts refer to documentation sources and could be the index cards of a card-and-cabinet system. 


\section{Multimedia}

The term "multimedia" was first used in 1966 to refer to the combined use of music and light effects. In the 1990s, multimedia took on its current meaning. In Vaughan (1993), the following definition has been presented:

Multimedia is any combination of text, graphic art, sound, animation, and video that is delivered by computer. When you allow the user, the viewer of the project, to control what and when these elements are delivered, it is interactive multimedia. When you provide a structure of linked elements through which the user can navigate, interactive multimedia becomes hypermedia. (p. 3)

The sketch presented in figure 6 clearly indicates that Otlet was fully aware of the importance and potential usability of different "modern" communication facilities that existed in his time. As such, he considered devices such as the telephone, radio, television, early videotapes, and gramophone discs to be important carriers of knowledge. What is worth noting is that Otlet already envisioned that these devices should be combined and made accessible from a single point of use. The matrix presentation in the middle part of the figure illustrates that information sources (or devices) should be combinable with each other, depending on the user's needs and the availability of sources. Otlet's combination of different devices corresponds with the combined handling and use of (digitized) multimedia in current computer systems.

\section{Distributed Information Systems}

In addition to studying the organization of an index for the "universe of knowledge," Otlet was also dealing with the problems of how user requests that were made from a great distance could be dealt with, how documentation from several sources could be integrated, and how requested documentation could be presented to the user through a single interface. In figure 7, Otlet shows a network that connects human users at different locations. Each user is connected through a telecommunication line with a central information source (the central office) and has his or her own connection device (telephone) to interact with an administrator who works at a central "Document Super Centre." The user sends a documentation request to an administrator, who processes the request and sends the requested documentation to the user. Eventually, a projector or television device could be used for the presentation of the returned documentation. Such an approach requires a tremendous effort to collect and organize all knowledge in principal documentation offices that are connected to the central "Document Super Centre," where the global index, based on the UDC, is maintained.

However, a digitized counterpart of Otlet's proposal exists nowadays. The human administrators are replaced by computer servers, which run 


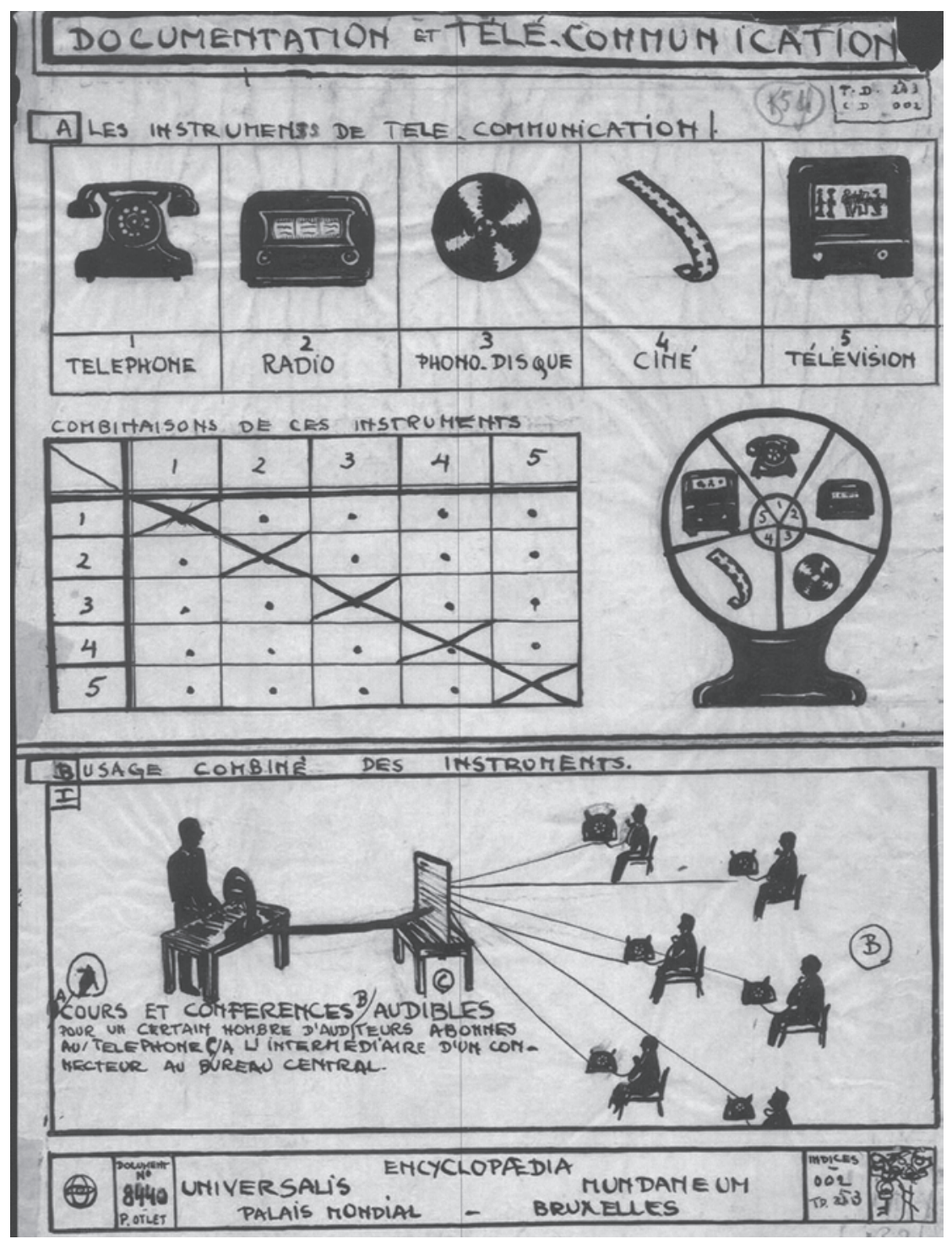

Figure 6. Instruments of telecommunication as seen by Paul Otlet (Mundaneum, EUM, Documentatio Universalis, doc nr. 8440).

database management systems that manage central (multimedia) databases. These computer servers are connected and distributed along a worldwide computer network: the Internet. Besides the data stored in such databases, a lot of information is also available in web pages, which are managed by web servers. Users can use their own computer devices to connect to the World Wide Web and use a web browser to connect and in- 


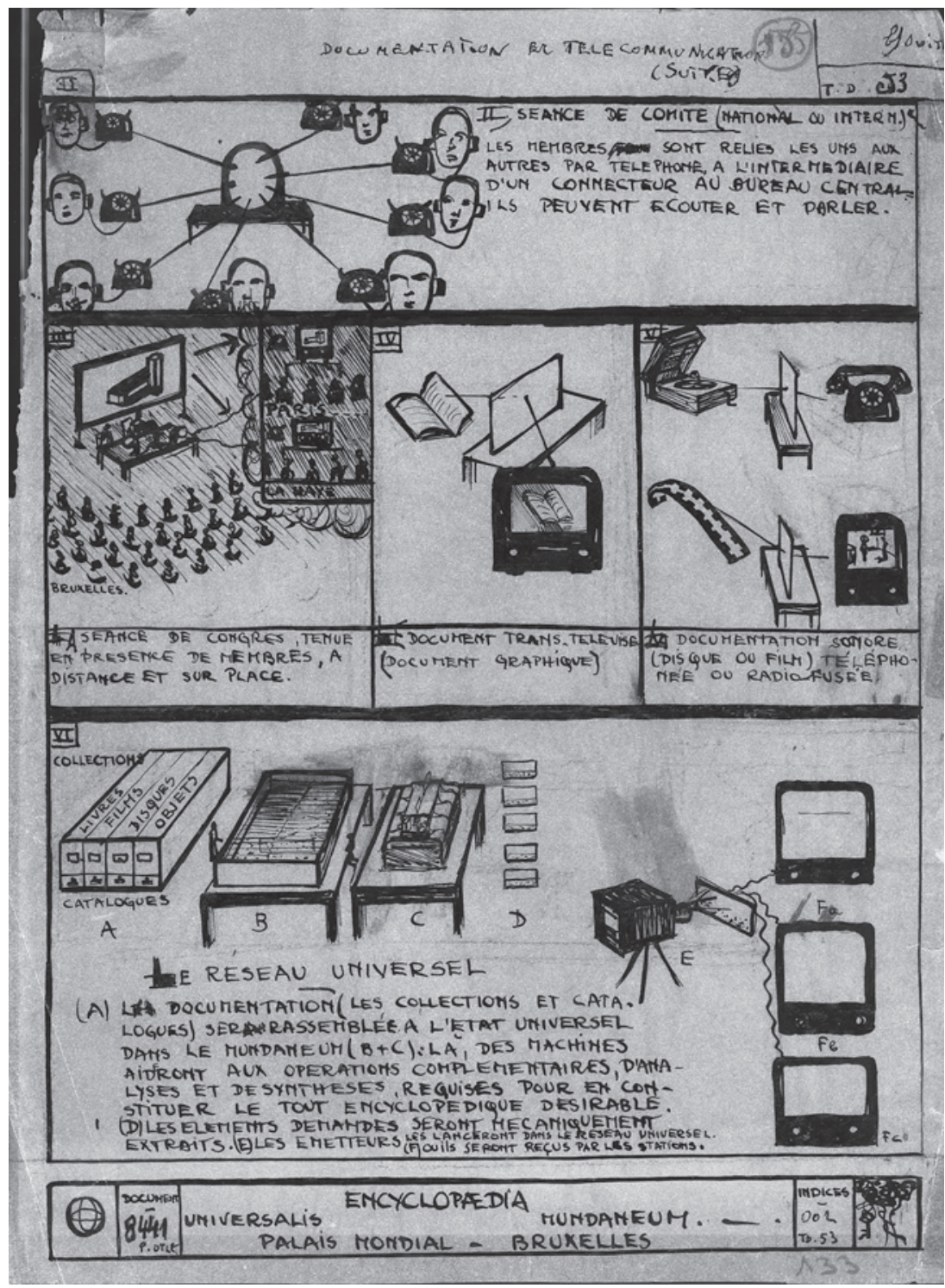

Figure 7. Documentation and telecommunication as seen by Paul Otlet (Mundaneum, EUM, Documentatio Universalis, doc nr. 8441, n 133).

teract with the accessible web and database servers. The development of the World Wide Web has a considerable impact on the development, use, and availability of multimedia. Unlike Otlet's proposal, the World Wide Web is not based on centrally governed policy for access and use but on heterogeneous interconnected information sources. 


\section{Open Problems and Challenges}

Despite the tremendous evolution of computer technology in recent years, computer scientists today still face some significant problems and challenges. Such challenges have been the driving forces behind new developments in information and knowledge management. In our work, we identified the following challenges that were also studied to some extent by Paul Otlet in the context of his knowledge spaces:

- How do we integrate data that originate from different, distributed data sources?

- How do we make information sources better accessible?

- How do we deal more efficiently with text and multimedia?

- How do we deal with imperfections in the data?

Next, each of these challenges is briefly described from the standpoint of digital spaces. Furthermore, we will discuss aspects of these challenges that were also considered by Otlet and, where possible, we will indicate how Otlet's ideas could be useful for related ongoing research.

\section{How Do We Integrate Data That Originate from Different, Distributed Data Sources?}

Information often originates from different, independent sources and can be stored at different locations on different information systems. Integrating this information in an accurate and efficient way remains a primary challenge. On the one hand, one can opt to keep the data where they are originally stored and maintain some kind of (hyper) links to connect related data. This approach is used in federated-database systems that consist of independent, interconnected databases that are stored at different systems in different locations. On the other hand, one can alternatively opt to merge the data from different sources into a single consistent representation of the data. This approach is used in central-database and data-warehouse systems. Both approaches have advantages and disadvantages and still face some challenges. In the linking approach, one has, among other things, to cope with the problems of data consistency (in the case of data replication, all replicas must at all times be consistent), distributed queries (there is no central system for handling queries), and visualization (query results from different sources must be integrated before being presented to the users). In the merging approach, one has, among other issues, to deal with automatic data extraction (from different sources), data transformation (to a common format), data correction (in case of errors), and data completion (in case of missing data). Moreover, a central system is more vulnerable to failures and can become a bottleneck if too many service requests are sent to it.

Otlet's Universal Network of Documentation dealt with the problem of how to organize all documentation in a practical manner and make it globally accessible in an efficient way. Otlet clearly opted for a centralized 
approach where principal documentation offices and libraries are connected to a central "Document Super Centre." Despite the current technological developments, such a central approach is not implementable in a practical manner on a worldwide scale, as it would require a lot of standardization, which in its turn would prevent local autonomy. Nevertheless, most computer scientists agree that a decentralized organization of information sources with restricted local autonomy, but organized in such a way that it looks to the users as if all information is centralized in one "virtual" source, would be a step in the right direction.

\section{How Do We Make Information Sources Better Accessible?}

Integrated, distributed information systems are very complex systems that handle huge quantities of heterogeneous data. It is still a real challenge to hide technical details of data management from the users of these systems and to make the stored information easily and efficiently accessible and searchable. Querying current databases involves the use of standardized query languages like SQL (American National Standard Institute, 1986). To use SQL, a user is required to understand the technical basics of relational databases. So, more flexible access techniques are required that are easier to understand and that support the use of natural language as spoken by the users. Such techniques might, for instance, be based on examples (query-by-example), support the use of linguistic terms in order to express user preferences (soft querying), make use of the user's profile (age, gender, profession, etc.) and context (when, where, etc.), or consider user feedback.

Otlet's solution for obtaining efficient access to documentation sources is to combine the UDC with a generic model that allows registered facts to be situated and their associated UDC codes to be found. One of these models is his cube model that we discussed earlier. Another model is his globe model, which is represented in figure 8 . In this model, a globe is considered to be a sphere that is composed of multiple rings. Each ring then corresponds with a dimension of the UDC. A globe can be embedded in another globe. A user can move the spheres by spinning them around the axis that connects the poles of the globe and by rolling them over the poles until the requested values (for each dimension under consideration) are positioned in the user's field of view. Otlet also considered different options to position the user: the user could be located in the core of the globe (i.e., inside the globe) or outside the globe. To our knowledge, the globe model has no counterparts in computer science, but its applicability as a data-organization, access, or visualization technique is definitely worth further investigation.

\section{How Do We Deal More Efficiently with Text and Multimedia?}

Querying, handling, and analyzing digitized multimedia are other challenges. Regular access techniques for multimedia collections are currently 


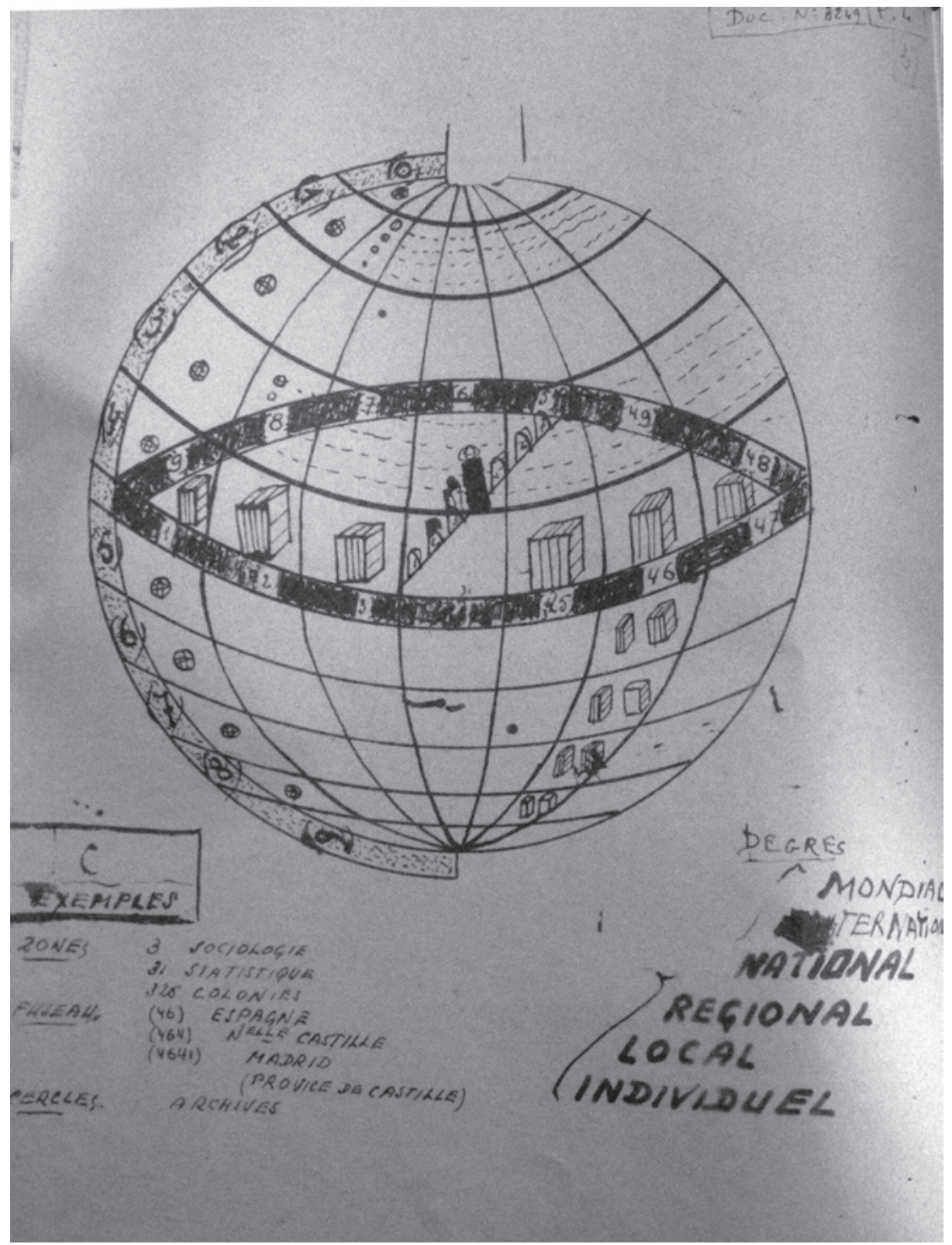

Figure 8. Paul Otlet's schema of (the organization of all knowledge of) the world (Mundaneum, Documentatio Universalis, doc nr. 8249).

based on querying the structured metadata that is associated with the media files. As such, it is, for example, possible to search for all photographs that were taken by a given person in July 2011. It is more interesting to search in multimedia collections on the basis of their content. Content-based querying facilities that support queries such as "find all 
photographs with an animal living in a green area," "find all audio recordings that sound similar to a given recording," or "find all video fragments where a red car is passing a black car" allow for fully exploiting multimedia collections. Recent pilot research proves the feasibility of contentbased querying, but much more research in this area is required. Also, full-text analysis and information retrieval in text documents are currently mainly based on keyword analysis. Recent research reveals that the extraction and analysis of semantically richer text elements like concepts and relationships between concepts allow for more efficient full-text processing and information-retrieval techniques.

Otlet's work on the UDC code might be relevant to recent research developments in full-text analysis. More specifically, the "concept-relationship-concept" patterns as specified earlier have recently been identified as semantically richer alternatives for keywords. To the best of our knowledge, only verbs (eventually combined with prepositions) have currently been considered as the relationships in such patterns. No further semantic classification on verbs has been considered so far. The UDC dimensions, which have been identified by Otlet as being important parameters for the classification of documentation, might be used to classify verbs and, as such, help to automatically find out what a text concerns. Further research on this topic is required.

Furthermore, as described here earlier, Otlet recognized the importance of combining different "modern" media for knowledge-exchange purposes long before than the term "multimedia" was invented. One such example is his proposal for the use of barometers-or what we would call today dashboard interfaces-to support decision making. Barometers as used in the stock exchange, Otlet suggested, could be compared to "a pair of scales of which the trays, when charged with new facts, directly go up or down, and determine the position of the needle" (Otlet, 1935, p. 419). Similarly, he thought it should be possible to create a system that anticipates and balances changes in our society. His "barometer of the world," shown in figure 9 , has three pointers, which indicate on a scale from zero to ten the recession, stability, or progress in the fields of economic, social, and political life. This barometer is surprisingly similar to the interactive dashboard interfaces used today. These are means for efficient humancomputer interaction and are composed of easily interpretable graphic elements like speedometers, traffic lights, or chart diagrams. An example of such a dashboard for rating, comparing, and evaluating customer satisfaction and retention in corporate and retail sales is shown in comparison with Otlet's "dashboard" in figure 9.

\section{How Do We Deal with Imperfections in the Data?}

Information, as provided by human beings, is often imperfect. Data imperfections are imposed by uncertainty and are mainly caused by the use 

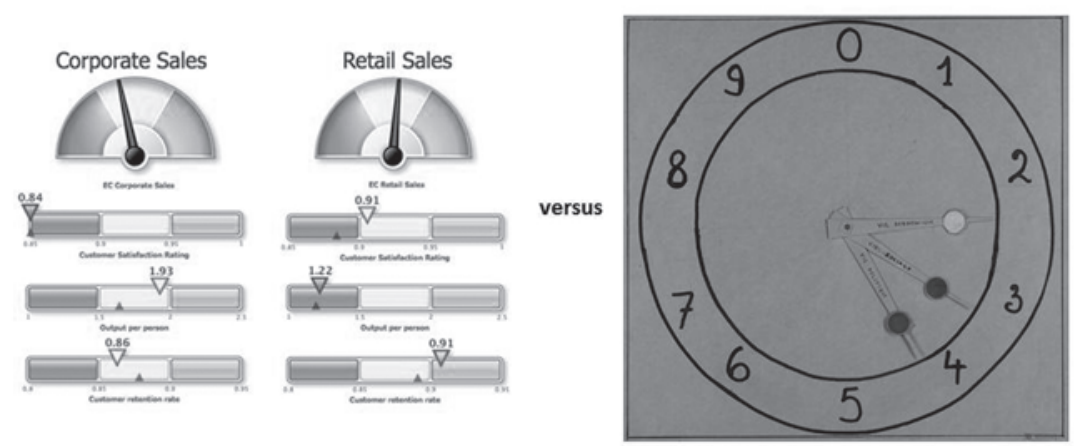

Figure 9. Paul Otlet's "Barometer of the World" versus a dashboard of corporate and retail sales (Mundaneum, EUM, fonds affiches, 00010848 ).

of natural language in human communication (vagueness), the lack of adequate measurement facilities (imprecision), or the unavailability of information (missing information). A categorization of data imperfections has been presented by Motro (1995). It is very important that imperfect data are modeled and handled as naturally and adequately as possible. If not, there is a heightened risk of errors, information loss, or inconsistencies in the data. This observation is one of the main driving forces behind the development of new conceptual, semantically richer data(base) models. Soft computing techniques (computing with linguistic variables), based on fuzzy set theory (Zadeh, 1965) and possibility theory (Dubois \& Prade, 1988), already provide basic facilities to handle imperfect information in a quite efficient way. Further research in this area is ongoing.

Otlet held a typical nineteenth-century positivistic view that ultimately all knowledge that is relevant and, hence, worth preservation is necessarily part of the great building of the sciences. Consequently, Otlet did not deal explicitly with imperfect knowledge, although he did recognize that imperfection should not be completely neglected. In fact, he explicitly considered the existence of "the Unknown" (what he called "l'Inconnu") in his knowledge spaces. This is among other things reflected by the fact that each ring in his globe model contains a special value or UDC dimension that represents a specific class for dealing with unknown values.

\section{Conclusions}

Computer science still faces the challenge of organizing, managing, and accessing very large digital collections of data, information, and knowledge in an efficient way. Computer systems can be seen as the main components of huge digital spaces in which all digitized knowledge is contained. 
In his search for mechanical solutions to the problem of efficiently organizing the world's documented knowledge in order to provide global access, Paul Otlet was faced with similar challenges to those faced by present-day computer scientists. Despite the differences in technology and approach, the knowledge spaces studied by Paul Otlet have more in common with modern digital spaces than one would at first glance expect. This makes it relevant and interesting to study Otlet's work and compare it with that of computers scientists today.

Our comparative study reveals that Otlet's vision of documentation and telecommunication has much in common with the World Wide Web and current multimedia applications. Moreover, it can be observed that Otlet's mechanical cube model was a true predecessor of the digital datawarehouse hypercubes that were(re)invented half a century later. Otlet further enhanced his mechanical cube model into a more general global model. Our study clearly reveals that it is useful to investigate Otlet's work in view of additional developments in computer science. Certainly, his global model and the applicability of the UDC for document and text analysis purposes in our opinion warrant continuing investigation not only to gain new insights into Otlet's work and thinking but also to study and understand their applicability and potential for organizing, handling, and exploring digital spaces more efficiently in the future.

\section{REFERENCES}

American National Standard Institute. (1986). The database language SQL. Document ANSI X3.135.

Codd, E. F. (1970). A relational model of data for large shared data banks. Communications of the ACM, 13(6), 377-387.

Dubois, D., \& Prade, H. (1988). Possibility theory. New York: Plenum Press.

Dunham, M. H. (2006). Data mining: Introductory and advanced concepts. Boston: Pearson Education.

Elmasri, R., \& Navathe, S. B. (2007). Fundamentals of database systems (5th ed.). Boston: Pearson Education.

Inmon, W.H. (1992). Building the data warehouse. New York: John Wiley \& Sons.

Kimball, R., \& Rose, M. (2002). The data warehouse toolkit. (2nd ed.). New York: John Wiley \& Sons.

Korth, H. F., \& Silberschatz, A. (1997). Database research faces the information explosion. Communications of the ACM, 40(2), 139-142.

Motro, A. (1995). Management of uncertainty in database systems. In Modern database systems: The object model, interoperability and beyond. Reading, MA: Addison-Wesley.

Otlet, P. (1907). L'Organisation systématique de la documentation et le développement de l'Institut International de Bibliographie. Brussels, Belgium: Institut International de Bibliographie.

Otlet, P. (1934). Traité de documentation: Le livre sur le livre, théorie et pratique. Brussels, Belgium: Editiones Mundaneum.

Otlet, P. (1935). Monde. Essai d'Universalisme. Connaissance du monde, sentiment du monde, action organisée et plan du monde. Brussels, Belgium: Mundaneum.

Otlet, P., \& Goldschmidt, R. (1925). La Conservation et la diffusion Internationale de la pensée: Le livre microphotique. Brussels, Belgium: IIB.

Vaughan, T. (1993). Multimedia: Making it work. (1st ed.). Berkeley, CA: Osborne/McGraw-Hill.

Zadeh, L. A. (1965). Fuzzy sets. Information and Control, 8(3), 338-353. 
Guy De Tré is associate professor in the Database, Document and Content Management research group of the Department of Telecommunications and Information Processing at Ghent University. He obtained the degree of doctor in engineering from Ghent University in 2000. His scientific interest is focused on data integration, the improvement of database accessibility, and flexible database querying, with special attention paid to the handling of imperfect information. This research was also the subject of his $\mathrm{PhD}$ thesis and has been validated by around seventy publications recorded in the Science Citation Index (including more than twenty book chapters, about forty contributions in the proceedings of international conferences, and three edited books). He is also the author of the book Principes van Databases, published by Pearson Education.

Wouter Van Acker is a postdoctoral researcher at the Department of Architecture and Urban Planning at Ghent University. He earned his civil engineering/architecture degree from Ghent University in 2004. For his doctoral dissertation (PhD, civil engineering/architecture, Ghent University, 2011), he studied the visionary schemes and schemas of the Belgian internationalist and encyclopedist Paul Otlet (1868-1944). His current research focuses on the spatial organization, representation, and dissemination of information and knowledge in urban settings. 